Ann. Biol. anim. Bioch. Biophys., I97r, 11 (2), I9I-Ig8.

\title{
EFFET DE L'ABSENCE D'INFORMATIONS OPTIQUES ET DE CONTACT PHYSIQUE SUR LA MANIFESTATION DES RELATIONS HIÉRARCHIQUES CHEZ LES BOVINS DOMESTIQUES
}

\author{
Marie-France BOUISSOU \\ avec la collaboration technique de Colette LAvenet et P. Orgeu \\ Station de Physiologie de la Reproduction, \\ Centre de Recherches de Tours, I. N.R. A., \\ $3 y$ - Nouzilly

\section{RÉSUMÉ}

Toutes les paires possibles d'un groupe de huit génisses, dont les relations sociales étaient connues, ont été soumises à des épreuves de compétition alimentaire de 3 minutes.

Les animaux de chaque paire ont été mis en présence cinq fois dans chacune des situations suivantes :

- témoins : les animaux se voyant et pouvant entrer en contact;

- les deux animaux étant privés d'informations optiques;

- les animaux ne pouvant entrer en contact physique.

Les résultats ont montré que la privation d'informations optiques n'empêche pas l'extériorisation des relations hiérarchiques. Par contre, la suppression de la possibilité du contact physique la perturbe complètement. Par ailleurs, le rôle de la reconnaissance individuelle dans le maintien des relations sociales est suggéré.

\section{INTRODUCTION}

Une organisation hiérarchique des groupes de Bovins domestiques a été mise en évidence par de nombreux auteurs (Schein, Fohrman, I955; GuHI, ATKESON, I959; Beillharz, MYlREA, I963 ; BoursSou, I965 ; Hook et al., I965 ; PorzIG, I965 ; DICKSON et al., I967; BRANTAS, I968). Les relations de dominance-subordination existant entre les animaux s'extériorisent, soit par des manifestations de type agressif : luttes, coups, menaces, soit par la soumission passive des animaux dominés qui s'écartent à l'approche d'un dominant et lui cèdent le pas lorsqu'il y a compé- 
tition. Dans un grand nombre de cas, il n'y a donc pas de contact physique entre les animaux, et des signaux agissant à distance interviennent seuls.

L'étude de ces signaux est importante pour comprendre le mécanisme d'établissement et de maintien des relations sociales. Dans cette étude, nous avons cherché à préciser le rôle respectif des informations optiques et du contact physique, lors de la manifestation des relations de dominance-soumission.

\section{MATÉRIEL ET MÉTHODES}

\section{A. - Les animaux}

Cette étude a porté sur huit génisses de race Frisonne Pie Noire, âgées de 3 ans, entretenues ensemble en stabulation libre depuis l'âge de 6 mois.

Une centaine d'heures d'observations, effectuées au début de l'expérience, a permis de connaître les relations existant entre tous les animaux.

Ces génisses étaient habituées aux manipulations par les expérimentateurs et familiarisées avec les dispositifs expérimentaux et la situation de compétition.

\section{B. - Épreuves de compétition}

Nous avons utilisé une épreuve de compétition alimentaire à laquelle sont successivement soumises toutes les paires possibles du groupe. Une telle épreuve facilite l'extériorisation des relations existant entre les animaux (Bovissou, 1970).

Les 28 paires constituées à partir des 8 animaux expérimentaux ont été réparties de telle sorte qu'un animal ne soit utilisé qu'une fois par jour et avec un seul partenaire; l'ordre dans lequel un animal donné rencontrait les autres était déterminé au hasard. Les animaux de chaque paire ont été mis en présence cinq fois dans chacune des situations suivantes :

- témoin : les animaux se voyant et pouvant entrer en contact ;

- les deux animaux étant privés d'informations optiques;

- les deux animaux ne pouvant entrer en contact corporel.

Les épreuves avaient lieu dans un parc de $30 \mathrm{~m}^{2}$ proche de celui où vivaient habituellement les génisses, le matin entre $9 \mathrm{~h}$ et II $\mathrm{h}$, les animaux étant à jeun depuis la veille.

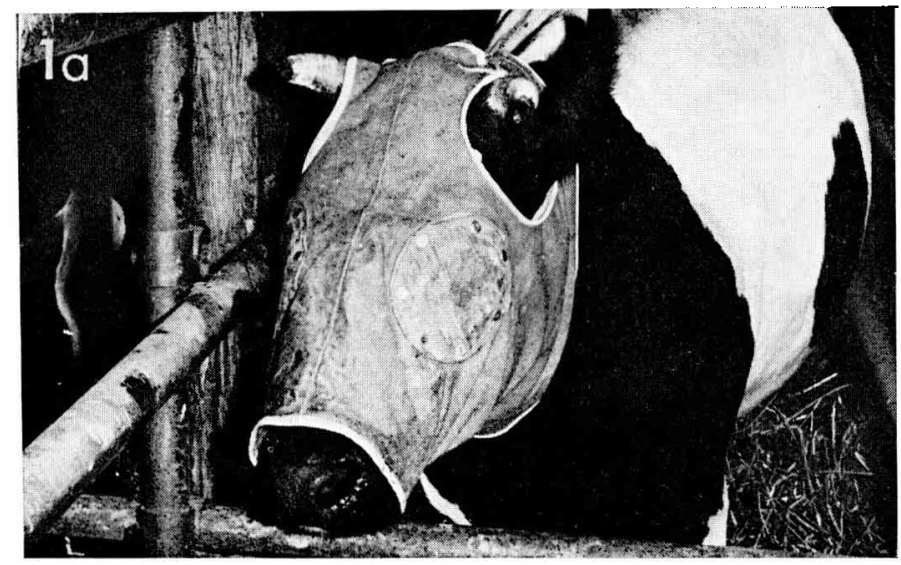

Fig, I.

a) Masque utilisé pour la privation d'informations optiques 


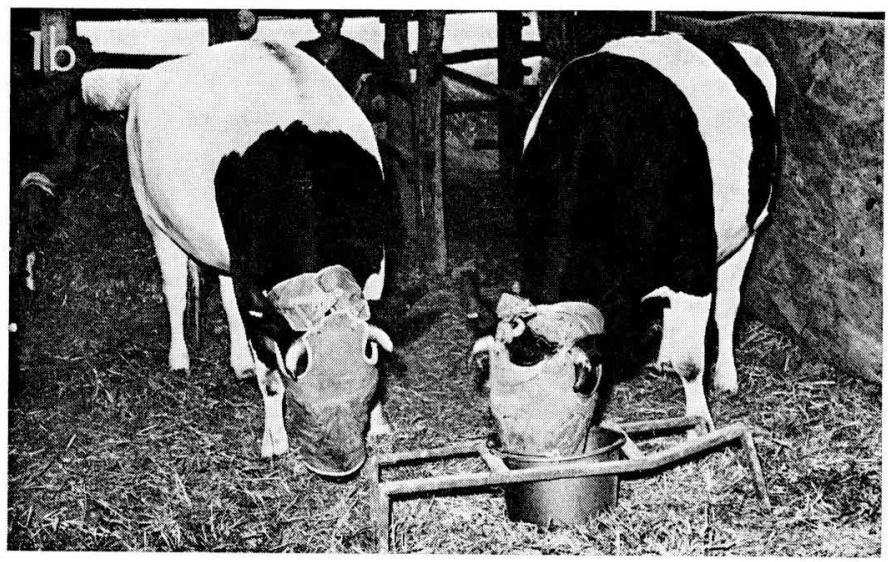

FIG. I

b) Épreuve de compétition alimentaire, les animaux étant privés d'informations optiques

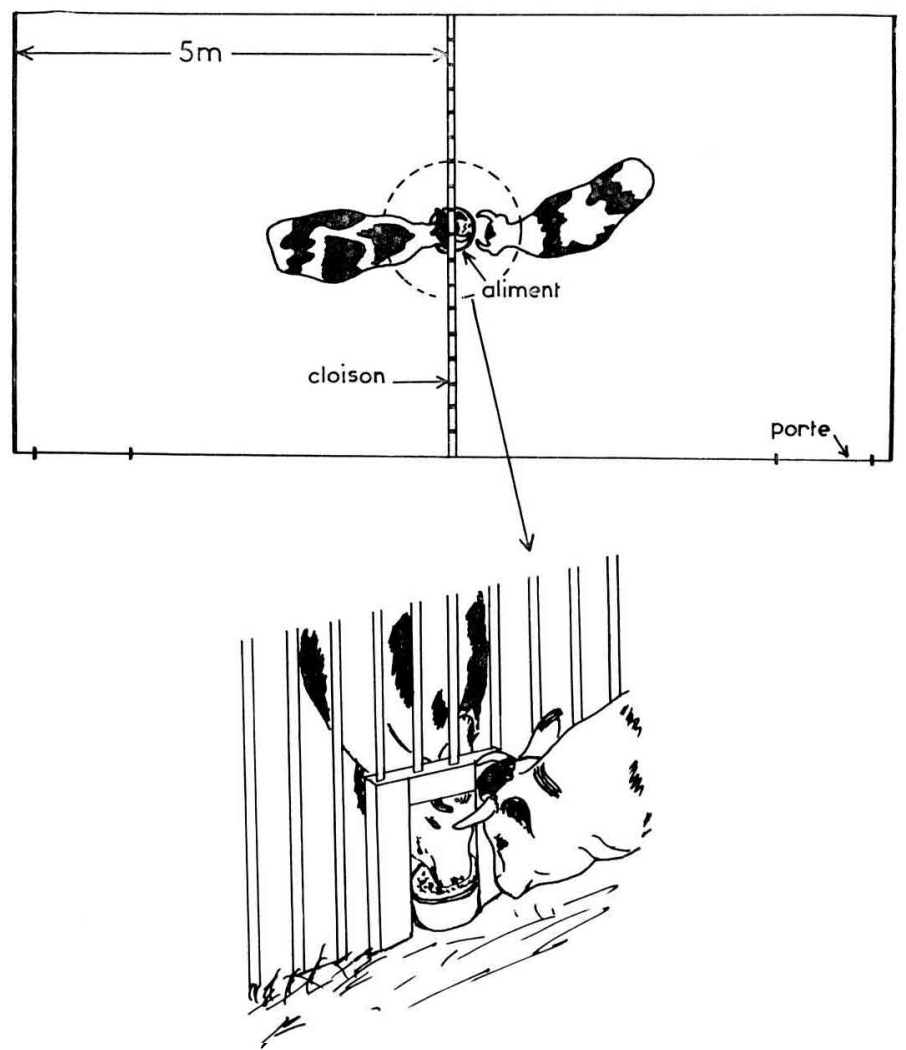

FIG. 2. - Schéma du dispositif utilisé pour les épreuves sans contıìt phı̀sıqü 
La suppression des informations optiques a été réalisée en faisant porter aux animaux un masque d'épaisse toile couvrant entièrement la tête à l'exception de l'extrémité du mufle (fig. I $a$ ).

Les animaux étaient conduits dans un couloir de contention où le masque leur était appliqué ; ils devaient ensuite sortir du couloir pour accéder au parc d'expérience. Chaque génisse a été individuellement habituée à cette situation. Au cours de l'expérience, on apportait l'aliment lorsque les deux vaches masquées étaient entrées dans le parc de test.

Lors des épreuves sans contact physique, le parc était divisé en deux parties par une cloison constitué de barreaux verticaux ; au centre et en bas de ce dispositif était pratiquée une ouverture de $40 \times 40 \mathrm{~cm}$ où se trouvait fixé le seau d'aliment. Les animaux étaient placés de part et d'autre de la cloison, ils pouvaient donc recevoir des informations optiques et olfactives mais ne pouvaient échanger de stimuli tactiles que par l'extrémité du mufle (fig. 2).

Dans les trois situations expérimentales, le temps d'alimentation de chaque animal était chronométré au cours d'une épreuve de 3 minutes; celle-ci débutait lorsque tous deux étaient également proches du seau, ce qui se produisait spontanément dans la majorité des cas. Lorsqu'au contraire la vache dominée refusait de s'approcher malgré l'intervention de l'expérimentateur, l'épreuve débutait une minute après l'introduction de l'aliment.

\section{RÉSULTATS}

Les résultats des épreuves témoins confirment ceux obtenus précédemment (Bouissou, I970) : dans tous les cas, la dominante de chaque paire s'alimente seule pendant les 3 minutes (tabl. I).

\section{TABLEAU I}

Comparaison entre les épreuves de compétition alimentaire "Témoins" avec privation d'informations optiques et avec privation de contact physique

\begin{tabular}{|c|c|c|c|}
\hline & Témoins & $\begin{array}{c}\text { Privation } \\
\text { d'informations } \\
\text { optiques }\end{array}$ & $\begin{array}{l}\text { Privation } \\
\text { du contact physique }\end{array}$ \\
\hline $\begin{array}{l}\text { Moyenne des temps d'alimentation } \\
\text { des dominantes de chaque paire/ } 3 \mathrm{mn}\end{array}$ & $3 \mathrm{mn}$ & $2 \mathrm{mn} 22 \mathrm{~s}$ & $1 \mathrm{mn} 49 \mathrm{~s}$ \\
\hline $\begin{array}{l}\text { Moyenne des temps d'alimentation } \\
\text { des dominées de chaque paire/3 mn }\end{array}$ & 0 & $46 s$ & $1 \mathrm{mn} 50 \mathrm{~s}$ \\
\hline $\begin{array}{c}\text { Nombre de paires où les dominées } \\
\text { ont eu accès à l'aliment au moins } 10 \mathrm{~s} \\
\text { Nombre d'épreuves } / 140\end{array}$ & $\begin{array}{l}0 \\
-\end{array}$ & $\begin{array}{l}23 \\
86\end{array}$ & $\begin{array}{r}28 \\
140\end{array}$ \\
\hline $\begin{array}{l}\text { Nombre de paires où les deux vaches } \\
\text { ont mangé simultanément au moins } 10 \mathrm{~s} \\
\text { Nombre d'épreuves } / 140\end{array}$ & $\begin{array}{l}0 \\
-\end{array}$ & $\begin{array}{l}25 \\
31\end{array}$ & $\begin{array}{r}28 \\
140\end{array}$ \\
\hline
\end{tabular}

Lorsque les animaux sont privés de vue, nous n'avons plus une réponse de type tout ou rien; alors que précédemment les vaches dominées ne s'étaient jamais alimentées, elles ont mangé dans cette situation au moins Io secondes dans 23 paires sur 28 ; dans 4 paires, la dominée a même, au cours de cartaines épreuves, mangé plus que la dominante. Cependant, la moyenne des temps d'alimentation des animaux dominants de chaque paire reste nettement supérieure à celle des animaux dominés (tabl. I). 
Lorsque les animaux ne peuvent entrer en contact physique, les moyennes des temps d'alimentation des dominantes et des dominées sont égales; dans toutes les paires, et au cours de toutes les épreuves, la dominée a mangé en même temps que la dominante. Dans presque la moitié des cas, elle a mangé plus (tabl. I).

Alors que précédemment, nous avions envisagé le temps moyen d'alimentation des dominantes et des dominées de chaque paire, nous pouvons calculer le temps moyen d'alimentation de chaque animal opposé à tous les autres, afin d'avoir une estimation de la situation au sein du groupe (fig. 3).

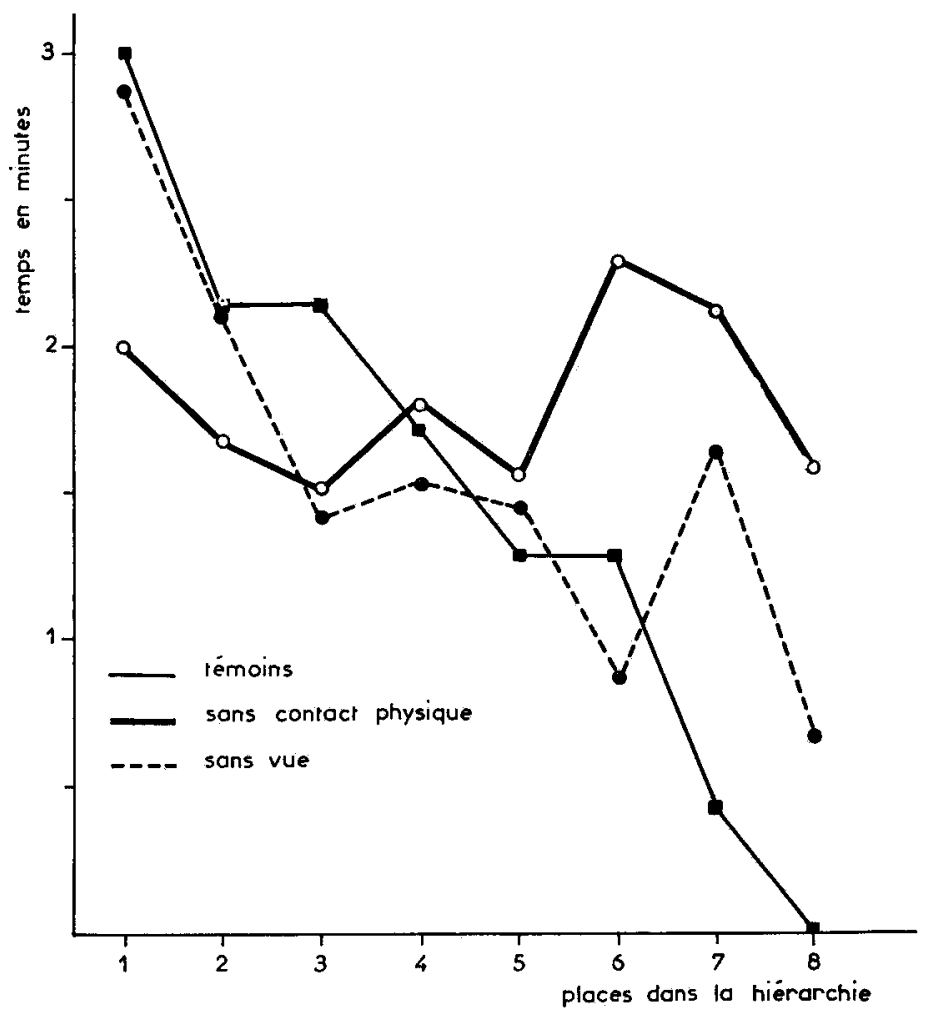

FIG. 3. - Temps d'alimentation moyen de chaque animal opposé à tous les autres en fonction du rang hiérarchique

Dans le cas des épreuves témoins, le temps d'alimentation moyen de 1'animal $\alpha$ du groupe est de 3 minutes, celui de l'animal $\omega$ est nul; les temps d'alimentation des autres animaux se répartissent dans un ordre décroissant correspondant à l'ordre hiérarchique. A deux reprises cependant, nous avons des temps égaux dus au fait que les animaux considérés étaient ex-aequo quant au nombre d'animaux dominés, la hiérarchie du groupe n'étant pas linéaire. Lorsque les génisses sont privées d'informations visuelles, les temps d'alimentation suivent encore assez bien l'ordre hiérarchique, alors qu'ils sont absolument sans rapport avec lui lorsque les animaux ne peuvent entrer en contact physique. 
Même au cours des épreuves où les animaux sont privés de la vue, on observe un certain nombre de manifestations agressives qui sont, comme celles survenant au sein du groupe, unidirectionnelles et conformes aux relations sociales existant entre eux. Dans ces conditions, les évincements sont très semblables à ceux observés lors des épreuves témoins, qu'ils résultent d'un léger coup donné avec une seule corne (fig. $4 a$ et $4 b$ ) ou d'un coup plus violent pouvant même être suivi d'un début de poursuite.
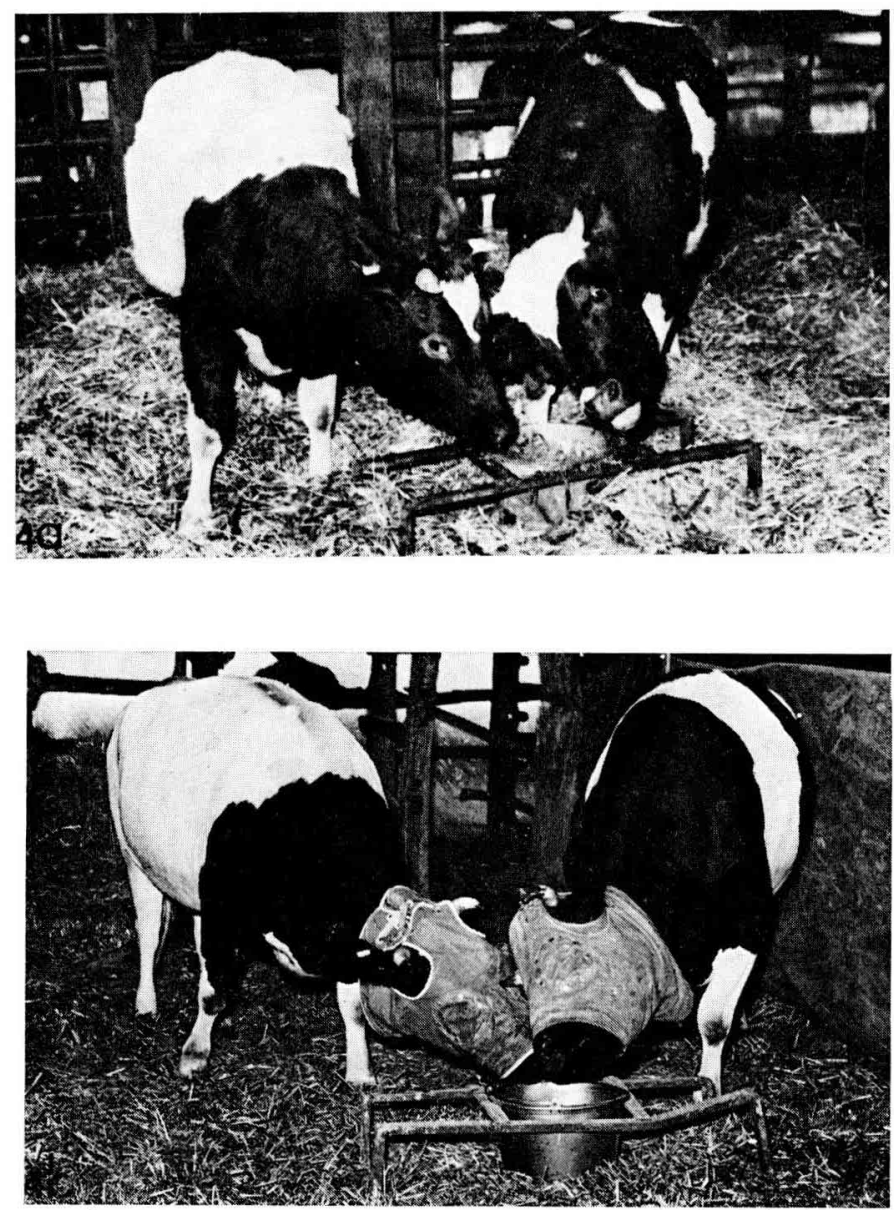

FIG. 4. - Evincement de l'animal dominé (à gauche) par l'animal dominant (à droite)

a) Lors des épreuves témoins

b) Lors des épreuves au cours desquelles les animaux sont privés d'informations optiques

Dans le cas où les animaux ne peuvent entrer en contact physique, lorsque les génisses dominées s'alimentent, les dominantes essaient de les évincer, mais elles ne peuvent que les repousser du mufle et ne parviennent pas à les éloigner définitivement ; des attitudes de menace sont fréquemment enregistrées, mais il est important de remarquer que dans ces conditions elles perdent toute efficacité. 


\section{IISCUSSION E'T CONCIUSION}

Les relations hiérarchiques une fois établies étant principalement maintenues par de simples menaces de l'animal dominant, ou par la soumission passive du dominé, il semblait légitime d'attribuer un rôle important aux informations optiques ; le contact physique, au contraire, semblait jouer un rôle moindre. Or, les résultats précédents montrent que la suppression de la vue n'empêche pas l'extériorisation des relations hiérarchiques.

Au cours des épreuves témoins, ou avec privation d'informations optiques, le rôle minime supposé joué par le contact physique semblait se confirmer : en effet, la vache dominée ne recevait souvent aucun coup dans la mesure où elle ne tentait pas de s'alimenter; néanmoins elle se tenait à distance. Or nous avons pu constater que l'interposition d'une cloison, interdisant le contact physique entre les animaux, perturbe totalement la manifestation des relations hiérarchiques. En effet, ni l'odeur de la dominante, ni ses attitudes de menace ne suffisent à interdire à la dominée l'accès du seau. Si le contact physique lui-même n'est pas nócessaire, il doit donc cependant être possible.

La manifestation des relations de dominance-soumission chez les Bovins n'obéit pas à un schéma rigide, une menace ou la simple vue de l'animal dominant provoquant la fuite du dominé. La suppression de la possibilité du contact physique entraîne poux ce dernier la disparition immédiate de la réaction d'évitement. Ces résultats diffèrent de ceux obtenus chez le Singe, où dans une situation analogue la seule présence du dominant empêche l'animal dominé de s'alimenter, bien qu'aucune sanction ne soit possible (WARDEN et GALT, I943).

Par ailleurs, nos résultats mettent en lumière le rôle important de la reconnaissance individuelle dans le maintien de la hiérarchie sociale ; en effet, lors des épreuves au cours desquelles les vaches sont privées de vue, la dominée s'abstient le plus souvent de s'alimenter et évite l'adversaire ; elle est donc informée de la présence d'un animal qui lui est hiérarchiquement supérieur. Les signaux sonores audibles étant inexistants, il semble que cette information ne puisse être que de nature olfactive. Comme nous pouvons difficilement admettre qu'elle porte sur la " valeur hiérarchique " elle-même, puisque cette valeur est essentiellement relative, il s'agit donc vraisemblablement dans ce cas d'une reconnaissance individuelle à base olfactive, à laquelle est liée une connaissance de la position hiérarchique de l'adversaire. Ces hypothèses restent à démontrer expérimentalement.

Ceci ne signifie naturellement pas que la vue ne joue aucun rôle ; dans les conditions normales il est probable que les différentes informations sensorielles agissent simultanément, cependant en l'absence d'une ou plusieurs d'entre elles, un comportement adapté peut néanmoins s'organiser. 


\section{SUMMARY \\ EFFECT OF PRECLUDING SIGHT OR PHYSICAL CON'TACT ON RANK ORDER BEHAVIOUR IN DOMESTIC CATTLE}

All possible pair groupings of 8 heifers with known social rank were submitted 5 times to each of the following $3 \mathrm{mn}$ competitive feeding tests :

I. The animals could see each other and come into contact during feeding (control test).

2. The animals were precluded from seeing each other.

3. The animals were precluded from physical contact.

For test I (control), the average feeding times were $3 \mathrm{mn}$ for the dominant animal vs. o for the subordinate. For test 2 , the average feeding times were $2 \mathrm{mn} 22 \mathrm{~s}$ vs. $46 \mathrm{~s}$ for test 3 , the average feeding times were identical ( $\mathrm{I} \mathrm{mn} 49 \mathrm{~s}$. vs. I $\mathrm{mn} 50 \mathrm{~s}$ ).

Deprivation of sight therefore does not hinder rank order behaviour, though in this case the feeding pattern was not total food vs. no food. On the contrary, precluding the possibility of physical contact totally abolishes the dominant vs. subordinate behaviour.

Our findings give supplemental evidence for the effect of individual recognition on the maintenance of rank order.

\section{RÉFÉRENCES BIBLIOGRAPHIQUES}

Beilharz R. G., Mylrea P. J., 1963. Social position and behavior of dairy heifers in yards. Anim. Behav., 11, 522-527.

Brantas G. C., I 968 . On the dominance order in Friesian Dutch dairy cows. Z. Tierzïcht. Ziuchtgsbiol., 84, I27-I 5 I.

Bourssou M. F., I965. Observations sur la hiérarchie sociale chez les Bovins domestiques. Ann. Biol. anim. Biochim. Biophys., 5, 327-339.

Bouissou M. F., I970. Technique de mise en évidence des relations hiérarchiques dans un groupe de Bovins domestiques. Rev. Comp. Anim., 4, 66-69.

Dickson D. P., BARR G. R., WIEckert D. A., I967. Social relationships of dairy cows in a feed lot. Behaviour, 29, 195-203.

Guhl A. M., Atrkeson F. W., 1959. Social organization in a herd of dairy cows. Trans. Kans. Acad. Sci., 62, 80-87.

Hook S. L., Donaldson S. L., Albright J. L., r965. A study of social dominance behavior in young cattle. Amer. Zoologist., 5, 7I4.

Porzig E., I965. Vorläufige Ergebnisse zum Einfluss der Sozialen Rangordnung auf das Verhalten des Hausrindes. Karl Marx Univ. Kolloquium, 25-26/3/I965.

Schein M. W., Fohrman M. H., 1955. Social dominance relationships in a herd of dairy cattle. Brit. J. Anim. Behav., 3, 45-55.

WARDEN C. J., Galt W., I943. A study of cooperation, dominćnce, grooming and other social tactors in monkeys. J. Genet. Psychol., 63, 213-233. 